\title{
Pneumocephalus after a Lumbar Epidural Steroid InJection
}

\author{
Anthony H. Guarino, MD, and Neill M. Wright, MD
}

Lumbar epidural steroid injections are a routine therapeutic procedure provided by pain management physicians. Despite a known low morbidity, significant acute events can occur.

Lumbar epidural steroid injections are a routine therapeutic procedure provided by pain management physicians. Despite a known low morbidity, significant acute events can occur.

\section{Case Report}

A 67-year-old female with a history of prior back surgery ( $\mathrm{L}_{3-4}$ microdiscectomy), hypertension, peripheral vascular disease, obesity, and DJD presented with sciatica. Her current medications included amitriptyline, propoxyphene, and diclofenac. This 5'4", 205 lbs. woman had an unremarkable physical exam. Her blood pressure was 150/80, heart rate 95 and regular, respiration rate 18 and $\mathrm{O}_{2}$ SATs of $93 \%$ on room air. Her MRI revealed multilevel DDD and spinal stenosis. An unremarkable lumbar epidural steroid injection was performed.

The epidural space was located under fluoroscopy at $\mathrm{L}_{4-5}$ with the loss of resistance technique using $2 \mathrm{~mL}$ of air. No blood or cerebrospinal fluid was aspirated before the injection of $6 \mathrm{~mL}$ of medication (2 mL 1\% lidocaine, $1 \mathrm{~mL} 80 \mathrm{mg}$ DepoMedrol, $3 \mathrm{~mL}$ normal saline).

From: Department of Anesthesiology, Pain Management Center, Washington University School of Medicine; and Department of Neurological Surgery, Washington University School of Medicine, St. Louis, MO

Address Correspondence:

Anthony H. Guarino, MD, 969 N. Mason Road,

Suite 240, St. Louis, MO 63141

Disclaimer: There was no external funding in prepa-

ration of this manuscript.

Conflict of Interest: None

Acknowledgement:

Manuscript received on 2/4/2005

Revision submitted on 3/9/2005

Accepted for publication on $3 / 11 / 2005$
We report a patient who developed symptoms mimicking a stroke after an epidural injection, and provide an evaluation and assessment of this patient plus other rare complications that occur with epidural

injections.

Keywords: Pneumocephalus, dural puncture, epidural steroid injection, post dural puncture headache

Twenty minutes later, while in the recovery room, the patient noted numbness in her tongue and mouth, sharpness behind her right eye, and generalized weakness. She had headache symptoms that were aggravated by movement of her head and were not relieved when lying supine. Her blood pressure was 180/100, heart rate 95 with normal sinus rhythm, respiration rate 18 and $\mathrm{O}_{2}$ SATs of $98 \%$ on room air. Her physical exam in the recovery room was unremarkable.

In the recovery room the patient was monitored continuously and no specific interventions were given. Within the first 15 minutes the patient's symptoms decreased. She was subsequently transferred to the emergency room where a head CT scan was performed and a pneumocephalus was diagnosed (Figs. 1 and 2). She was discharged home and her symptoms completely resolved over the ensuing week. No specific interventions were given.

The constellation of acute diffuse neurological changes in a middle aged obese female with known peripheral vascular disease initially caused concern for an evolving stroke; the signs resembled strokes previously witnessed by the authors. Although the epidural was uneventful, the procedure can produce high levels of stress, possibly enough to catalyze a stroke in a patient with high risk factors. Fortunately, during assessment of the patient, these concerns were decreased because the acute changes began resolving immediately. However, symptoms still remained, so she was transferred to the emergency department for a full work-up, including a head CT that demonstrated pneumocephalus. A similar sequence of events had not occurred before in the roughly 10,000 epidurals performed by the authors. Therefore, a case report on pneumocephalus and other potential complications was undertaken to share these findings with colleagues.

\section{DisCussion}

Possible complications that can occur as a result of an epidural injection include dural puncture, vascular injection, and subarachnoid hemorrhage. Symptoms of these complications include acute headache, neurological changes, cardiovascular and respiratory changes, and sudden onset of back and leg pain.

An acute onset headache after a lumbar epidural injection is a symptom that can be associated with a postdural puncture headache (PDPH), a migraine, a subarachnoid hemorrhage (SAH), or a pneumocephalus. A careful history and physical will help clarify the cause. PDPH generally produces a positional headache with an onset 24-48 hours after a dural puncture. A migraine is recurrent and symptoms are typically similar with each headache. SAH is extremely rare but has evolving symptoms and is lethal $50 \%$ of the time; prompt neurosurgical treatment is required (1). When air is used in the loss of resistance technique, pneumocephalus should be considered. Pneumocephalus headaches are typically not position dependent. A CT scan will help differentiate the diagnosis of SAH and pneumocephalus (2).

\section{Accidental Injection of Air \\ A pneumocephalus can be caused by air entering the meninges during a proce-}




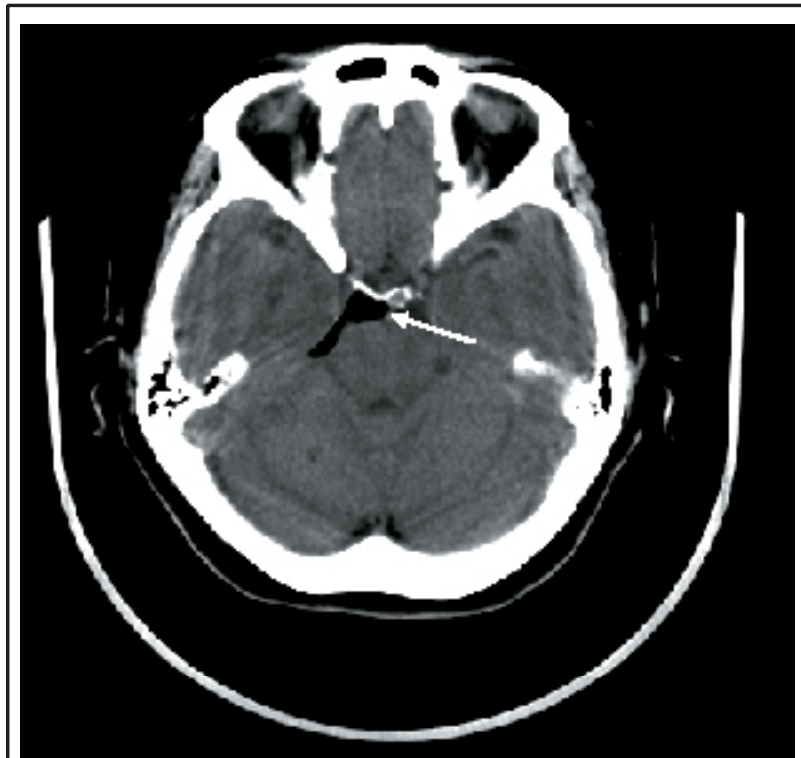

Fig. 1. Axial CT scan windowed for soft tissue, revealing pneumocephaly in the prepontine cistern on the right (arrow)

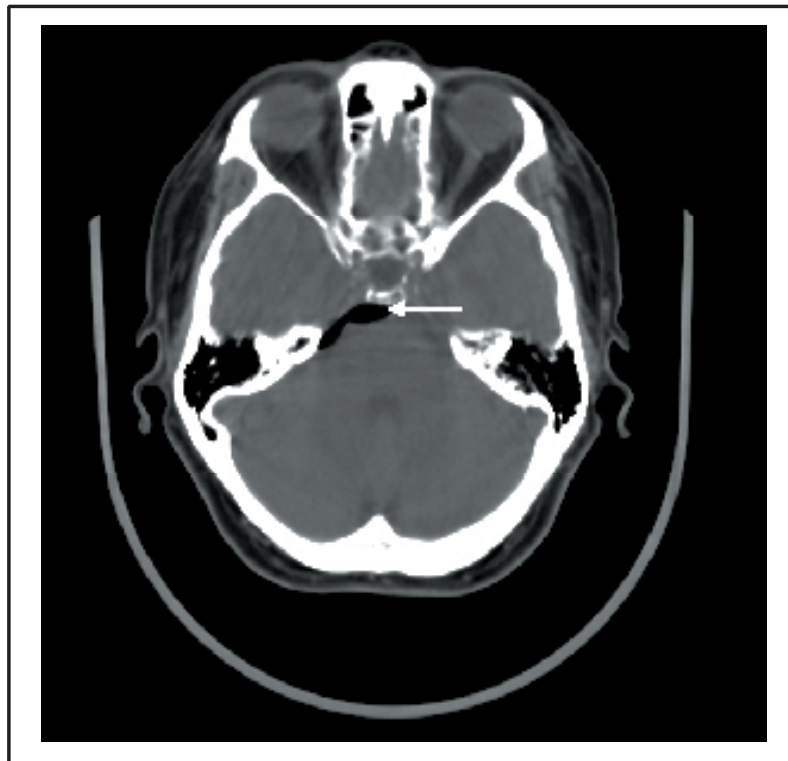

Fig. 2. Axial CT scan windowed for bone, revealing pneumocephaly in the prepontine cistern on the right (arrow) dure, either by injection or by the pressure differential created between the spine and an unoccluded needle. A pneumocephalus typically causes a sudden headache (3); it may manifest symptoms and signs ranging from a persistent headache, lethargy, confusion, and slow arousal, to hemiparesis and hemiplegia (4). The symptoms usually resolve over one week, concomitant with the reduction of cranial air (4). Neurologic complications can occur whether or not air is used in the loss of resistance technique $(5,6)$. Sudden headache accompanied by associated neurologic deficits after a spinal procedure suggests the diagnosis of pneumocephalus.

In the above case report, the patient's one week recovery was consistent with reported rates of absorption of air (7-9). Use of a high inspired air/oxygen concentration can hasten absorption of intracranial air collection (10). Increasing the inspired oxygen concentration reduces the partial pressure of nitrogen in the blood, creating a greater concentration gradient for nitrogen, the main constituent of the intracranial air collection, to diffuse into the blood stream (11). Long-term sequelae from pneumocephalus have not been reported in the literature.

Although rare, and typically associated with intracranial endoscopic procedures or open craniotomies, tension pneumocephalus is a life-threaten- ing variation of pneumocephaly. In tension pneumocephalus, the volume of intracranial air places pressure on the brain similar to epidural hematomas and can cause herniation, and ultimately death, if not promptly recognized and treated with surgical evacuation of the trapped air pocket. Although unlikely, pneumocephaly resulting from lumbar procedures should be considered in the setting of severe headache followed by obtundation.

Pneumocephalus can occur whether the needle penetrates the dura or the arachnoid membrane; however, a subdural pneumocephalus may be more painful (12). The potential for a pneumocephalus can be reduced by using normal saline during the loss of resistance technique as well as limiting the time the stylet is withdrawn from the needle (2). Less than $2 \mathrm{ml}$ of subarachnoid air can cause headache (8). The volume of air that can be safely injected into the epidural space has not been established (13). The correlation between the amount of intracranial air and headache symptoms is less than perfect (8).

Pneumocephalus is a well-known complication of spinal and epidural anesthesia, but it is extremely rare after a lumbar epidural steroid injection. This can obscure the clinical diagnosis, leading to unnecessary procedures and prolonged patient discomfort (14).

\section{Other Accidental Injections}

In addition to pneumocephalus, intrathecal injections of medications can cause problems. An injection of a local anesthetic intrathecally can cause a central block that extends up into a high sensory level, endangering cardiovascular and respiratory stability. If these problems occur, blood pressure, heart rate, and respiration should be supported pharmacologically, and ventilation assisted as indicated. Although not substantiated in published literature, arachnoiditis has been reported as another complication of intrathecal steroids.

An unintentional injection of a large bolus of medication into a vascular structure during an epidural injection can lead to serious complications. Large doses of local anesthetics can cause neurologic problems as well as respiratory and cardiovascular compromise. Choosing the proper anesthetic agent and volume to accompany the steroid can reduce potential complications. Problems are not usually observed when $2 \mathrm{~mL}$ of $1 \%$ lidocaine is used. Besides limiting the amount of local anesthetic injected, a test dose of local anesthetic with epinephrine can be given to confirm extravascular status. In addition, fluoroscopic guidance is recommended for needle placement with spinal injections. Even with traditional interlaminar injections using the loss of resistance 
technique, nonionic contrast agent, such as iohexol, will help delineate the exact location of the needle tip, and aid in determining whether or not the needle tip is intravascular, subdural or intrathecal. Injections should be incremental, and all necessary equipment and drugs should be available to treat local anesthetic-induced systemic toxicity.

\section{Other Unintended Problems \\ Accidental dural puncture with a} large bore needle occurs approximately $1 \%$ of the time; dural puncture is the most commonly reported complication (15). Use of an 18- or 19-gauge needle results in a $50 \%$ chance of a headache developing (16). Higher caliber needles will yield lower incidences of headache. Initial therapy for the positional headache should include fluids with caffeine, bed rest, and analgesics. Recalcitrant headaches can be treated with a blood patch, which involves the injection of autologous blood into the epidural area at the site of the presumed puncture.

Epidural hematomas are extremely rare. A hematoma usually occurs in the setting of a coagulopathy or when using anticoagulants, however, a patient with no known risk factors can also develop a hematoma (17). Presenting symptoms usually involve back and leg pain. An emergent scan (CT or MRI) should be obtained to verify a suspicion. If not treated with decompression within eight hours or less, a hematoma pressing on a neurologic structure may lead to irreversible deficits.

Epidural infections can lead to abscesses, discitis, osteomyelitis, and/or meningitis. This occurrence, though rare, should be suspected if new back and/or leg symptoms occur after an injection. The combination of diabetes and steroid immunosuppression may predispose a patient to epidural abscess formation (18). Imaging and blood tests will help clarify the problem. Epidural infection usually arises endogenously from hematogenous spread, secondary to infection elsewhere in the body. S. aureus is the most common infection; antibiotic prophylaxis for S. aureus should be considered for immuno-compromised patients undergoing epidural corticosteroid injections (19). Any neurological changes should prompt an immediate neurosurgical consultation. Many infections can be managed conservatively.

Unintended neural damage can also occur during needle placement. If the needle has caused neural damage in the spinal canal, injection of the steroid can worsen the situation and should not be done. If there is any doubt as to the placement of the needle the procedure should be stopped. Subsequent care should include a neurological consultation, IV steroids, and palliative medications.

\section{CONCLUSION}

The case report presents a rare phenomenon of a pneumocephalus after an epidural injection. Physicians should consider this diagnosis when a patient reports an acute headache after an epidural injection. Diagnosis should be confirmed with a head CT. The clinical course is benign with resolution of the headache expected over a one-week period.

\section{Author Affiliation:}

Anthony H. Guarino, MD

Faculty, Department of Anesthesiology

Pain Management Center

Washington University School of

Medicine

969 N. Mason Road, Suite 240

St. Louis, MO 63141

E-mail: guarinoa@msnotes.wustl.edu

\section{Neill M. Wright, M.D.}

Assistant Professor

Department of Neurological Surgery

Washington University School of

Medicine

660 S. Euclid Avenue, Box 8057

St. Louis, MO 63110

E-mail: wrightn@nsurg.wustl.edu

\section{RefERENCES}

1. Vasdev GM, Chantigian RC. Pneumocephalus following the treatment of a postdural puncture headache with an epidural saline infusion. J Clin Anesth 1994; 6:508-511.

2. Kawamata T, Omote K, Matsumoto M, Toriyabe $M$, Ito $T$, Namiki A. Pneumocephalus following an epidural blood patch. Acta Anaesthesiologica Scandinavica 2003; 47: 907-909.

3. Ash KM, Cannon JE, Biehl DR. Pneumocephalus following attempted epidural anaesthesia. Can J Anaesth 1991; 38:772774 .

4. Katz Y, Markovits R, Rosenberg B. Pneumoencephalos after inadvertent intrathe- cal air injection during epidural block. Anesthesiology 1990; 73:1277-1279.

5. Aida S, Taga K, Yamakura T, Endoh H, Shimoji K. Headache after attempted epidural block: the role of intrathecal air. Anesthesiology 1998; 88:76-81.

6. Avellanal M, Olmedilla L, Ojea R, Rueda ML, Navia J. Pneumocephalus after spinal anesthesia. Anesthesiology 1996; 85:423425.

7. Abram SF, Cherwenka RW. Transient headache immediately following epidural steroid injection. Anesthesiology 1979; 50: 461-462.

8. Roderick L, Moore DC, Artru AA. Pneumocephalus with headache during spinal anesthesia. Anesthesiology 1985; 62:690692.

9. Kuczkowski KM. Pneumocephalus following an uneventful lumbar puncture: Does the gauge of a spinal needle matter? Anesth Analg 2004; 99:303-304.

10. Becker WJ. Pneumocephalus as a cause for headache. Can J Neurol Sci 2002; 29: 278-281.

11. Dexter F, Reasoner DK. Theoretical assessment of normobaric oxygen therapy to treat pneumocephalus. Anesthesiology 1996; 84:442-447.

12. Hogan QH, Haddox JD. Headache from intracranial air after a lumbar epidural injection: subarachnoid or subdural? Reg Anesth 1992; 17:303-305.

13. Saberski LR, Kondamuri S, Osinubi OY. Identification of the epidural space: is loss of resistance to air a safe technique? A review of the complications related to the use of air. Reg Anesth 1997; 22:3-15.

14. Kozikowski GP, Cohen SP. Lumbar puncture associated with pneumocephalus: report of a case. Anesth Analg 2004; 98:524526.

15. Abram SE, O'Connor TC. Complications associated with epidural steroid injections. Reg Anesth 1996; 21:149-162.

16. Brown DL. Atlas of Regional Anesthesia. 1st ed. WB Saunders Company, Philadelphia, 1992.

17. Stoll A, Sanchez M. Epidural hematoma after epidural block: Implications for its use in pain management. Surg Neurol 2002; 57:235-240.

18. Benzon HT, Iqbal M, Tallman MS, Boehlke L, Russell EJ. Superior sagittal sinus thrombosis in a patient with postdural puncture headache. Reg Anesth Pain Med 2003; 28:64-67.

19. Hooten WM, Kinney MO, Huntoon MA. Epidural abscess and meningitis after epidural corticosteroid injection. Mayo Clin Proc 2004; 79(5):682-686. 